\title{
Probing Weak Intermolecular Interactions in Self-Assembled Nanotubes
}

\author{
Laurent Bouteiller ${ }^{*,+\neq}$ and Paul van der Schoot ${ }^{*, \S, \perp}$ \\ ${ }^{\dagger}$ Chimie des Polymères, UMR 7610, UPMC Univ Paris 06, F-75005 Paris, France \\ ${ }^{\ddagger}$ Chimie des Polymères, UMR 7610, CNRS, F-75005 Paris, France \\ ${ }^{\S}$ Group Theory of Polymers and Soft Matter, Technische Universiteit Eindhoven, P.O. Box 513, 5600 MB Eindhoven, The \\ Netherlands \\ ${ }^{\perp}$ Instituut voor Theoretische Fysica, Universiteit Utrecht, Leuvenlaan 4, 3584 CE Utrecht, The Netherlands
}

Supporting Information

ABSTRACT: Extreme confinement affects the physical properties of fluids, but little quantitative data is available. We report on studies of a bisurea compound that self-assembles into nanotubes to probe solvent confinement on the angstrom scale. By applying a statistical model to calorimetric data obtained on solvent mixtures, we show that the thermodynamic stability of the nanotubes is an extremely sensitive function of the solvent composition because solvent interactions inside and outside of the nanotubes are different. We are able to measure energetic effects as small as $0.01 \mathrm{kT}$ and relate them to the differences in molecular structure of the solvents.
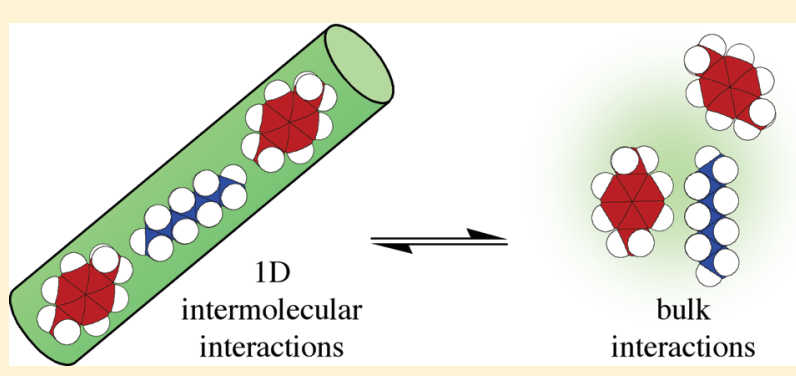

\section{INTRODUCTION}

Physical properties of fluids are known to change under the influence of confinement and more so the stronger the confinement: fluids in carbon nanotubes a few nanometers across exhibit lower friction than in micrometer-sized capillaries, ${ }^{1}$ solvent mixtures in contact with sufficiently thin capillaries fractionate, ${ }^{2}$ and phase transitions change order if trapped in Vycor glass if the pore size is small enough. ${ }^{3}$ This has ramifications for a wide variety of fields, including geology, ${ }^{4}$ biology, ${ }^{5-7}$ chemical engineering, ${ }^{8}$ and supramolecular chemistry. ${ }^{9}$ Still, little is known, let alone understood, about the effects of confinement on fluids in tubes that are so thin that only a single file of fluid molecules fit in. ${ }^{10}$ In this paper, we report on studies of a bisurea compound that self-assembles into nanotubes in a large number of solvents to probe solvent confinement on the angstrom scale. Self-assembled nanotubes are ideally suited for this, because their thermodynamic stability is an extremely sensitive function of the solvent composition. By applying a statistical model to experimental data obtained on twelve binary solvent mixtures, we find that solvent interactions inside the nanotubes are different from those in the bulk. For almost all mixtures tested, interactions between unlike solvent molecules are less favorable inside the nanotubes than outside. The coupling between nanotube self-assembly and intermolecular interactions with (and between) encapsulated solvent molecules also provides an unprecedented insight into how small differences in intermolecular interactions have a large impact on supramolecular assemblies and highlights the important role of solvent therein, a role that so far has not received the attention that it merits in the experimental and theoretical literature. ${ }^{11}$

The compound we focus attention on belongs to a class of bisureas known to self-assemble, through intermolecular hydrogen bonding, into filaments below a polymerization temperature $T^{*}$, and into narrow tubes for temperatures $T \leq$ $T^{* *} \leq T^{*} \cdot{ }^{12,13}$ While the polymerization of monomers into filaments is cooperative, the filament-tube transition at $T^{* *}$ is even sharper and resembles a phase transition. It is characterized by a peak in the heat capacity and can be pinpointed accurately as a function of concentration, solvent type, etc. ${ }^{14,15}$ An example of a state diagram is given in Figure 1. It turns out that the filament-tube transition is extremely sensitive to the solvent in which the bisureas are dissolved. The polarity of the solvent plays an important role, but more significant is the size and shape of the solvent molecules. ${ }^{16}$ Molecular modeling and host-guest studies have shown that the inner diameter of the self-assembled tubes measures at most $7 \AA$, suggesting there is barely enough space to allow for a single file of solvent molecules. ${ }^{16,17}$ Molecules wider than the optimal radius distort the self-assembled nanotube and hence destabilize it. Also, the longer the solvent molecules, the more orientational entropy is lost upon their encapsulation. Clearly, interactions between pairs of solvent molecules in- and outside a tube must also be different because confinement frustrates their optimal positioning.

Received: November 14, 2011

Published: December 16, 2011 

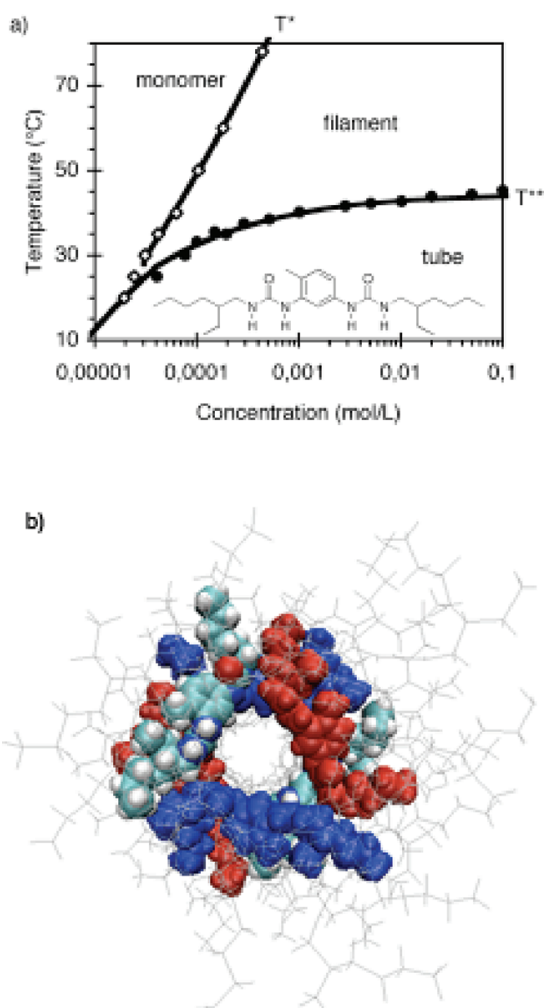

Figure 1. (a) Chemical structure of bisurea EHUT and its phase diagram in toluene. ${ }^{15}$ (b) Optimized nanotube structure determined by molecular mechanics and dynamics (six individual molecules are represented with a different color). ${ }^{13}$

By investigating how the filament-tube transition temperature varies with composition in a variety of solvent mixtures, and by applying a theoretical model that describes the impact of solvent on the stability of self-assembled nanotubes, we are able to probe extremely small changes in interaction free energies between the various components caused by the confinement. The competition between tube-solvent and solvent-solvent interactions explains the strongly nonlinear dependence of the filament-tube transition temperature $T^{* *}$ on the bulk solvent composition. Competition between self-assemblies amplifies small differences in interactions that otherwise cannot straightforwardly be measured nor obtained from computer simulation. $^{14}$

\section{RESULTS AND DISCUSSION}

Figure 2 shows differential scanning calorimetry (DSC) traces measured for bisurea EHUT solutions in toluene/1,3,5trimethylbenzene (TMB) mixtures. The endothermic transition is characteristic for the transition from the tube to the filament structure. For the twelve binary solvent mixtures studied, the heating and the cooling runs are symmetrical, confirming the reversibility of this transition. The transition temperature, $T^{* *}$, was taken at the maximum of the endothermic peak and is plotted versus solvent composition in Figures $3 a$ and S1 (Supporting Information). The variation is clearly nonlinear with a positive departure of as much as $10{ }^{\circ} \mathrm{C}$ from the expected linearity if the solvent inside and outside the tubes would behave identically. Of course, a change of solvent may affect the nanotube precise structure, which is expected to have a direct influence on the transition temperature. However, a conformational change induced by a direct solvent-nanotube

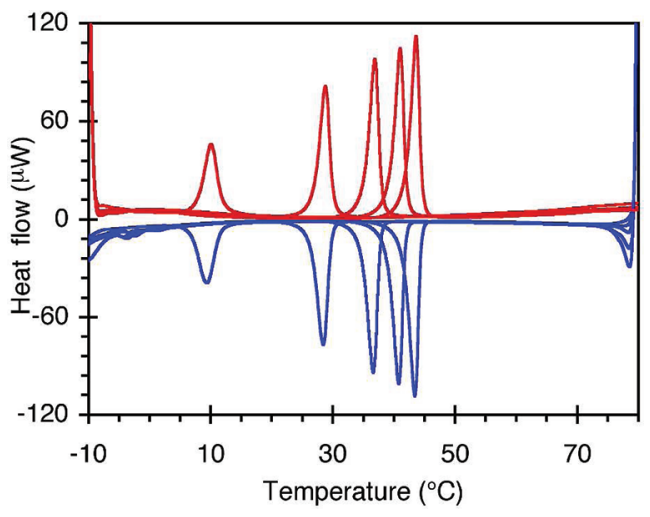

Figure 2. Calorimetric characterization of the tube to filament transition. DSC thermograms (heating in red; cooling in blue) for solutions of EHUT in toluene/1,3,5-trimethylbenzene (TMB) mixtures. The molar composition of the solvent is $0 / 100,21 / 79$, $43 / 57,70 / 30,100 / 0$, from left to right. $[\mathrm{EHUT}]=0.01 \mathrm{~mol} / \mathrm{L}$.

interaction affects only the portion of the tube in direct contact with the solvent molecule. Therefore, direct solvent-nanotube interactions cannot explain the nonlinearity observed.

An obvious explanation for the nonlinear behavior observed is fractionation of the solvent. The bisurea tubes are more stable in toluene than in TMB as judged from the higher $T^{* *}$; therefore, one expects the tubes to encapsulate toluene preferentially, implying its concentration inside the tubes to be larger than outside. This could explain the positive deviation of the curve. To test this hypothesis, we measured the composition dependence of the bisurea transition temperature for diverse solvent mixtures (Table 1). Remarkably, for some mixtures a negative deviation was measured, e.g., heptane and 1chloropentane (Figure 3c). If the negative deviation was the consequence of solvent fractionation, then this would mean that the tubes favor the solvent in which the tubes are the least stable. Arguably, this is unphysical and shows that solvent fractionation cannot be the sole reason for the nonlinear variation of the transition temperature.

To provide an explanation for our observations, we apply a statistical theory based on the law of mass action and a model for the interaction of the solvent mixture with the assemblies, described in the Supporting Information. There are seven model parameters, five of which are fixed by DSC of the solutions in the pure solvents 1 and 2 ; see Table 1 . These include the transition temperatures $T_{1}{ }^{*}$ and $T_{2} * *$ in the two solvents, and the difference between binding enthalpies $h_{1} \equiv h$ and $h_{2} \equiv h+\Delta h$ of single bisureas in tubes and in filaments. ${ }^{18}$ The difference of the solvent-contribution $\Delta \varepsilon$ to the excess free energy of binding of the monomers in a tube in the two types of solvent is also fixed by the pure solvent data, because the transition temperature difference $T_{2} * *-T_{1} * *$ is proportional to $\Delta \varepsilon .{ }^{19}$ Only two unknowns are left: a free energy $J$ and its associated enthalpy $h_{f}$, describing the effect of confinement on the interaction between the two types of solvent. If $J>0$, unlike contacts are penalized by the confinement in the nanotube while if $J<0$, the reverse is true.

Because $J \equiv h_{J}-T_{1}{ }^{*} s_{J}$, we for simplicity consider the enthalpy-dominated case where $s_{J}=0$ and the entropydominated one where $h_{J}=0$. Hence, in the curve fitting to our experimental data we have a single adjustable parameter, $J$, and examine the two cases $h_{J}=0$ or $h_{J}=J$. Figure $3 \mathrm{a}$ and $3 \mathrm{c}$ shows curve fits for two solvent mixtures. Irrespective of whether $h_{J}=$ 
14
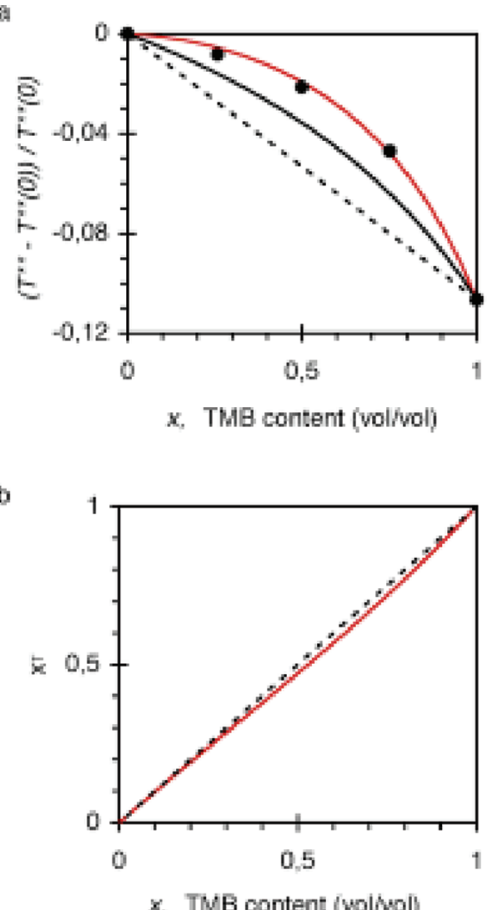

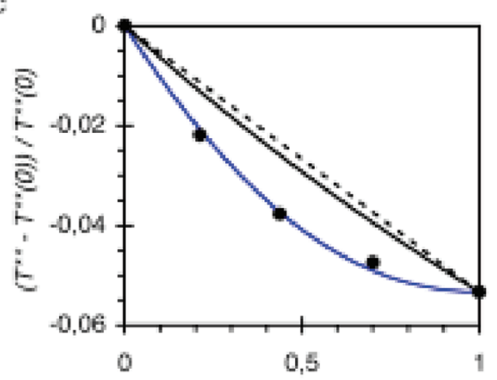

x, 1-chibropentane content (volkvol)

d

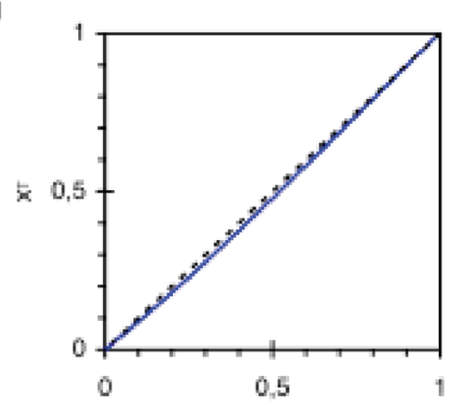

$x$, 1-chibropentane content (vikifoli)

Figure 3. Influence of bulk solvent composition on tube stability and on solvent composition in the tubes. Normalized transition temperature ( $\left.T^{* *}\right)$ versus bulk solvent composition $(x)$ for EHUT in toluene/TMB mixtures (a) or heptane/1-chloropentane mixtures $(c)\left(T^{* *}(0)=T_{1} * *\right.$ and $\left.T^{* *}(1)=T_{2} * *\right)$. Drawn lines: curve fit with $J=h_{J}=-0.025$ (a: red), $J=h_{J}=0.016$ (c: blue), or $J=h_{J}=0$ (black). Calculated volume fraction of TMB (b) or 1-chloropentane (d) in the tubes versus bulk fraction.

Table 1. Values for the Parameters Deduced from the Composition Dependence of $T^{* *}$ for EHUT in Mixed Solvents (compositions probed: approximately $0 / 100,25 / 75,50 / 50,75 / 25$, and $100 / 0$ )

\begin{tabular}{|c|c|c|c|c|c|c|c|c|c|}
\hline entry & solvent 1 & solvent 2 & $T_{1} * * a\left({ }^{\circ} \mathrm{C}\right)$ & $T_{2} * * a\left({ }^{\circ} \mathrm{C}\right)$ & $h^{b}\left(k_{\mathrm{B}} T\right)$ & $\Delta h^{c}\left(k_{\mathrm{B}} T\right)$ & $\Delta \varepsilon^{d}\left(k_{\mathrm{B}} T\right)$ & $T \Delta s^{e}\left(k_{\mathrm{B}} T\right)$ & $f^{f}\left(k_{\mathrm{B}} T\right)$ \\
\hline 1 & toluene & TMB & 43.5 & 9.8 & -2.0 & 0.9 & 0.12 & 0.8 & -0.025 \\
\hline 2 & $p$-xylene & TMB & 49.0 & 9.8 & -2.0 & 0.9 & 0.13 & 0.8 & -0.020 \\
\hline 3 & octane & heptane & 75.4 & 72.9 & -1.5 & 0.2 & 0.01 & 0.2 & 0.001 \\
\hline 4 & tert-butylbenzene & $p$-xylene & 50.9 & 49.0 & -2.1 & 0.1 & 0.01 & 0.1 & 0.001 \\
\hline 5 & tert-butylbenzene & toluene & 50.9 & 43.5 & -2.1 & 0.1 & 0.05 & 0.1 & 0.002 \\
\hline 6 & $p$-xylene & toluene & 49.0 & 43.5 & -2.0 & 0.0 & 0.03 & 0.0 & 0.004 \\
\hline 7 & tert-butylbenzene & TMB & 50.9 & 9.8 & -2.1 & 1.1 & 0.14 & 0.9 & 0.010 \\
\hline 8 & heptane & $p$-xylene & 72.9 & 49.0 & -1.4 & -0.6 & 0.14 & -0.8 & 0.010 \\
\hline 9 & heptane & 1,9-decadiene & 72.9 & 65.9 & -1.4 & -0.1 & 0.03 & -0.2 & 0.010 \\
\hline 10 & heptane & toluene & 72.9 & 43.5 & -1.4 & -0.6 & 0.17 & -0.8 & 0.015 \\
\hline 11 & heptane & 1-chloropentane & 72.9 & 54.4 & -1.3 & -0.3 & 0.08 & -0.4 & 0.016 \\
\hline 12 & heptane & TMB & 72.9 & 9.8 & -1.4 & 0.3 & 0.20 & 0.1 & $0.035(0.04)$ \\
\hline
\end{tabular}

${ }^{a}$ Transition temperature for EHUT, measured by DSC; uncertainty $\pm 0.2{ }^{\circ} \mathrm{C}$. ${ }^{b}$ Transition enthalpy for EHUT in solvent 1 , measured by DSC; uncertainty $\pm 0.15 k_{\mathrm{B}} T$. ${ }^{c}$ Difference in transition enthalpy for EHUT in solvents 1 and $2\left(\Delta h=h_{2}-h_{1}\right)$, measured by DSC; uncertainty $\pm 0.3 k_{\mathrm{B}} T$. ${ }^{d}$ Excess free energy; uncertainty $\pm 0.03 k_{\mathrm{B}} T$. ${ }^{e}$ Uncertainty $\pm 0.33 k_{\mathrm{B}} T .{ }^{f}$ Interaction parameter between solvent 1 and 2 , deduced by fitting the DSC data of mixtures, assuming that $h_{J}=J .{ }^{g}$ Interaction parameter between solvent 1 and 2 , deduced by fitting the DSC data of mixtures, assuming that $h_{J}=0$.

0 or $J$, a near perfect fit is obtained, suggesting our results are robust albeit that we cannot discriminate between enthalpic and entropic confinements effects.

The curve with a positive deviation (Figure 3a) gives a good agreement with a negative value for $J$, while the curve with a negative deviation (Figure 3c) gives a positive value for $J$. We applied our model to the data obtained for all 12 solvent mixtures investigated: all of them can be fitted accurately, requiring a single adjustable parameter $J$. The numerical values that we find (Table 1) are mere fractions of the thermal energy, yet setting $J=0$ does not describe the data adequately (see Figure $3 \mathrm{a}$ and $3 \mathrm{c}$ ). As previously, setting $h_{J}$ equal to 0 or $J$ has no discernible effect, except for mixtures of the solvents heptane and TMB. For this single case, we therefore have a larger uncertainty for $J$ : $0.035 \leq J / k_{\mathrm{B}} T \leq 0.04$. Still, the robustness of the model is remarkable; all the data can be explained on the basis of the strength of interactions between two unlike solvent molecules confined in one spatial dimension relative to that in three. Apparently, unlike contacts are penalized under confinement for all mixtures, except TMB/ toluene and TMB $/ p$-xylene. That the magnitude of $J$ we find is small, at most $0.04 k_{\mathrm{B}} T,{ }^{20}$ seems reasonable, given that the solvents selected do not possess strongly interacting groups and in bulk seem to behave like ideal mixtures. ${ }^{21}$

Despite this, it is possible to detect a clear trend in our results. The four mixtures in which the smallest interaction 
energy is found $\left(|J| \leq 0.004 k_{\mathrm{B}} T\right)$ involve similar solvents: two linear alkanes (entry 3 ) or two aromatic hydrocarbons with no ortho nor meta substituent (entries 4 through 6). All the mixtures for which $|J| \geq 0.01 k_{\mathrm{B}} T$ concern less symmetrical systems: (i) a linear alkane and an aromatic hydrocarbon (entries 8, 10, and 12); (ii) a linear alkane and an unsaturated or chlorinated alkane (entries 9 and 11); (iii) an aromatic hydrocarbon with para substituents and an aromatic hydrocarbon with meta substituents (entries 1, 2, and 7). The correlation between the value of the interaction parameter $J$ and the similarity of solvent molecules suggests that the main physical effect is captured by our model.

Table 1 also presents our findings for the difference between the solvent contributions to the excess free energy of binding of the monomer in a tube, $\Delta \varepsilon$, and its breakdown in the contributions from the enthalpy $\Delta h$ and entropy, $T \Delta s=\Delta h-$ $\Delta \varepsilon$. The enthalpies are up to 10 times larger than the free energies. This shows the important role that solvent entropy plays in the driving force for the supramolecular assembly of molecules.

We now return to the fractionation of the solvents in- and outside the nanotubes. Results of our calculations in Figure $3 \mathrm{~b}$ and $3 \mathrm{~d}$ show that it must be insignificant, at most a few percent. This is to be expected because the interaction free energies we find are small; see Table 1. Also, the quasi-1D character of the solvent in the tubes makes it sensitive to fluctuations that diminish any tendency toward fractionation. This confirms that fractionation cannot explain our findings and must be due to the effects of confinement on the interactions between the solvent molecules in the tubes.

\section{CONCLUSION}

In conclusion, the interaction free energy between neighboring solvent molecules placed in a single file configuration, caused by confinement in a nanotube, differs from that in a bulk mixture. Although the difference is very small for all mixtures tested, we conclude that the larger the difference in the molecular structure of the solvent molecules, the larger the influence of confinement on the net interaction between them. In most but not all mixtures, interactions between unlike species are penalized under conditions of extreme confinement. It is not clear why this is so, and hopefully our experiments inspire (ab initio) calculations of intermolecular interactions and help increase our understanding of the consequences of extreme confinement on molecules.

\section{ASSOCIATED CONTENT}

\section{S Supporting Information}

Experimental procedures and description of the theoretical model. This material is available free of charge via the Internet at http://pubs.acs.org.

\section{AUTHOR INFORMATION}

\section{Corresponding Author}

laurent.bouteiller@upmc.fr; p.p.a.m.v.d.schoot@tue.nl

\section{ACKNOWLEDGMENTS}

P.v.d.S. is grateful to UPMC for their hospitality and financial support.

\section{REFERENCES}

(1) Falk, K.; Sedlmeier, F.; Joly, L.; Netz, R. R.; Bocquet, L. Nano Lett. 2010, 10, 4067-4073.

(2) Gelb, L. D.; Gubbins, K. E.; Radhakrishnan, R.; SliwinskaBartkoviak, M. Rep. Prog. Phys. 1999, 62, 1573-1659.

(3) Iannacchione, G. S.; Crawford, G. P.; Zumer, S.; Doane, J. W.; Finotello, D. Phys. Rev. Lett. 1993, 71, 2595-2598.

(4) Mallamace, M.; Corsaro, C.; Broccio, M.; Branca, C.; GonzalezSegredo, N.; Spooren, J.; Chen, S.-H.; Stanley, H. E. Proc. Natl. Acad. Sci. U.S.A. 2008, 105, 12725-12729.

(5) Wang, H.-J.; Xi, X.-K.; Kleinhammes, A.; Wu, Y. Science 2008, $322,80-83$.

(6) Acharya, R.; Carnevale, V.; Fiorin, G.; Levine, B. G.; Polishchuk, A. L.; Balannik, V.; Samish, I.; Lamb, R. A.; Pinto, L. H.; DeGrado, W. F.; Klein, M. L. Proc. Natl. Acad. Sci. U.S.A. 2010, 107, 15075-15080.

(7) Duan, C.; Majumdar, A. Nat. Nanotechnol. 2010, 5, 848-852.

(8) Sozzani, P.; Bracco, S.; Comotti, A.; Ferretti, L.; Simonutti, R. Angew. Chem., Int. Ed. 2005, 44, 1816-1820.

(9) Rebek, J. Jr. Angew. Chem., Int. Ed. 2005, 44, 2068-2078.

(10) Hahn, K.; Kärger, J.; Kukla, V. Phys. Rev. Lett. 1996, 76, $2762-$ 2765.

(11) Jonkheijm, P.; van der Schoot, P.; Schenning, A. P. H. J.; Meijer, E. W. Science 2006, 313, 80-83.

(12) Bouteiller, L.; Colombani, O.; Lortie, F.; Terech, P. J. Am. Chem. Soc. 2005, 127, 8893-8898.

(13) Shikata, T.; Nishida, T.; Isare, B.; Linares, M.; Lazzaroni, R.; Bouteiller, L. J. Phys. Chem. B 2008, 112, 8459-8465.

(14) Roman, M.; Cannizzo, C.; Pinault, T.; Isare, B.; Andrioletti, B.; van der Schoot, P.; Bouteiller, L. J. Am. Chem. Soc. 2010, 132, 1681816824.

(15) Bellot, M.; Bouteiller, L. Langmuir 2008, 24, 14176-14182.

(16) Pinault, T.; Isare, B.; Bouteiller, L. Chem. Phys. Chem. 2006, 7, 816-819.

(17) Isare, B.; Linares, M.; Lazzaroni, R.; Bouteiller, L. J. Phys. Chem. B 2009, 113, 3360-3364.

(18) All free energies, enthalpies, and entropies are tacitly understood to be at the temperature $T=T_{1}$ **.

(19) See details in Supporting Information.

(20) A thermal energy unit $\left(k_{\mathrm{B}} T\right)$ corresponds at room temperature to about $2.5 \mathrm{~kJ} / \mathrm{mol}$.

(21) Kehiaian, H. V. Landolt-Börnstein Group IV Physical Chemistry Vol. 23 Binary Liquid Systems of Nonelectrolytes; Springer: New York, 2009. 